\title{
The Screening Method of a Bifidogenic Dietary Fiber Extracted from Inedible Parts of Vegetables
}

\author{
Emiko IWATA, Hisako HoTTA and Masahiro Goтo \\ Faculty of Home Economics, Kobe Women's University, 2-1 Aoyama, Higashisuma, \\ Suma-ku, Kobe 654-8585, Japan \\ (Received March 9, 2009)
}

\begin{abstract}
Summary Total dietary fiber (DF) was extracted from the inedible parts of vegetables such as peel of taro and Chinese yam, pea pod, broad bean pod, and broad bean testa. Effects of these fibers on the growth of bifidobacteria were determined by two kinds of experiments: one was to determine the increase of Bifidobacterium longum JCM1217 (B. longum) in medium containing DF, the other was an in vitro fermentation of the DF by anaerobic slurries of mixed human fecal or rat cecal microbiota. Anaerobic culture was carried out for $48 \mathrm{~h}$ in both experiments. In the pure culture of B. longum, the significant increase of bacterial number was observed as compared with $0 \mathrm{~h}$ in the medium containing the DF from peel of Chinese yam, pea pod and broad bean pod $(p<0.05)$. On the other hand, bacterial number was decreased in the medium containing the DF from the peel of taro and broad bean testa. We selected the DF from pea pod because of its highest bifidogenic property in human fecal microbiota. In the fermentation of DF from pea pod by rat cecal microbiota, bifidobacteria and lactobacilli were increased. Bacteroidaceae and clostridia were decreased. These results suggested that DF from pea pod had possibility as a prebiotic. The bifidogenic property was affected by the sugar composition of DF.
\end{abstract}

Key Words dietary fiber, bifidobacteria, lactobacilli

It is well known that the human colon is the most populated region for gut bacteria. More than 500 kinds of bacteria with the number of $10^{12}$ for every gram of feces exist in the human colon (1). Those compositions of bacteria vary with individuals.

Lactic acid-producing bacteria (LAB) such as lactobacilli and bifidobacteria are health promoting, having the activities of resistance to pathogenic bacteria, lowering blood ammonia, increased stimulation of the immune response and a reduction of cancer (1). Dietary modulation of the human gut microflora by functional foods such as probiotics and prebiotics is mainly designed to increase LAB.

Probiotics are live bacteria such as LAB. Prebiotics are non-digestible carbohydrates such as dietary fiber (DF) which are selectively fermented by beneficial bacteria. A convincing prebiotic is an inulin or inulintype fructan (2). Other non-digestible oligosaccharides with reported prebiotic activity include galacto-, isomalto-, soybean-, xylo-, and gentio-oligosaccharides, lacturose, and lactosucrose (3).

This study aims at a screening of DF which is fermented by a Bifidobacterium longum. Before beginning an in vivo study, focus on the most bifidogenic DF in vitro is needed to save time. The reason for using the inedible parts of vegetables is that DF must be highly concentrated in them. In addition, as their structural

E-mail: emiko.i@suma.kobe-wu.ac.jp analyses have not been published yet, novel DF may be found. Until now, there are several reports concerning the food by-products. Fermentability of apple (4) or orange pomace (5) and coffee waste (6) have been published and the bifidogenic property was only observed in the DF from coffee waste. DF has two forms, water-soluble and water-insoluble, and the physiological effects are reported to be different. Only water-soluble DF has been reported to be a suitable substrate for DF. But both types of DF have a bulking effect, which results in an increased fecal output (7). Therefore, in this report, we used both types to clarify the prebiotic effect because of the possibility of new functional foods.

\section{Materials and Methods}

Materials. Pea pod (from Wakayama), broad bean pod (from Kagoshima), broad bean testa (from Kagoshima), peel of taro (from Ehime) and Chinese yam (from Hokkaido) were selected as inedible parts of vegetables. To take a peel from taro and Chinese yam, a peeler was used. Broad beans were boiled, and then the testa was taken off. After lyophilization, the dried samples were milled smaller than $0.8 \mathrm{~mm}$. DF were extracted by the Prosky method (8).

Screening of bifidogenic dietary fibers by pure culture of bifidobacteria. Bifidobacterium longum JCM1217 were purchased from Riken Bio Resource Center (Tsukuba, Japan) for the selection of bifidogenic DF. To clarify the bifidogenic property of the DF, lacturose, a known pre- 
biotic, was chosen as a positive control. One percent of peptone/yeast extract medium (PY medium) was prepared for pre-culture. After the pre-culture, B. longum was cultured to $0.5 \%$ DF or lacturose containing $0.5 \%$ PY medium in which $1 \%$ DF suspension was mixed with $1 \%$ PY medium. Colonies were counted at $0 \mathrm{~h}$ and after $48 \mathrm{~h}$ by plating culture medium on GAM agar (Nissui, Tokyo, Japan).

Fermentation studies of dietary fibers with human fecal microbiota or rat cecal microbiota. Human feces were preliminarily obtained from a woman $(35 \mathrm{y})$ who had constipation. The samples were quickly transferred under anaerobic conditions, and immediately subjected to the examination. In one defecation, $1.5 \mathrm{~g}$ feces were weighed into $4.5 \mathrm{~mL}$ of anaerobic diluent. After stirring of those solutions, $0.5 \mathrm{~mL}$ was added to $0.5 \%$ PY medium containing $0.5 \%$ DF or lacturose. These media were cultured anaerobically at $37^{\circ} \mathrm{C}$ for $48 \mathrm{~h}$.

Four Wistar male rats (age $8 \mathrm{wk}$ ) were purchased from CLEA Japan, Inc. (Osaka, Japan). All animals and experimental protocols were approved by Ethical Committee of Kobe Women's University on Animal Research. The temperature $\left(22-24^{\circ} \mathrm{C}\right)$, humidity (50 $5 \%$ ), lighting (7:00 am to 7:00 pm) and air conditioning were controlled in the animal room. The rats were fed for $1 \mathrm{wk}$ with CE-2 standard rat chow (Oriental Yeast Co., Ltd., Tokyo, Japan) and tap water ad libitum. All animals were completely bled from the large abdominal vein under pentobarbital anesthesia (sodium pentobarbital $45 \mathrm{mg} / \mathrm{kg}$ ). Then the cecum was quickly dissected from the abdomen. Cecal contents were mixed thoroughly and $0.5 \mathrm{~g}$ was weighed into $4.5 \mathrm{~mL}$ of anaerobic diluents (9). After that, those were treated as same as human fecal microbiota.

The method for bacterial analysis after fermentation followed that of Mitsuoka et al. (10). With one exception, all experiments were carried out in a glove box saturated with nitrogen gas. Residual oxygen concentration was monitored with an oxygen gas meter OX-94G (Riken Keiki, Tokyo, Japan) (9).

A series of 10 -fold dilutions $\left(10^{-1}\right.$ to $\left.10^{-7}\right)$ was made in anaerobic diluents. From appropriate dilutions, $0.05 \mathrm{~mL}$ aliquots were spread onto agar plates which are indicated below. Glucose blood liver (BL) agar (Nissui) and MRS agar (DIFCO, Maryland, USA) were cultured anaerobically for $48 \mathrm{~h}$ at $37^{\circ} \mathrm{C}$ to detect bifidobacteria and lactobacilli in human fecal microbiota, and bifidobacteria, lactobacilli, bacteroidaceae and clostridia in rat cecal microbiota. The identification of bacterial group was performed with colonial and cellular morphologies, Gram staining, spore formation and aerobic growth.

Analysis of constituent sugars. Alditol acetylation was carried out, following the Englyst method (11). The sample was dissolved in chloroform. Then sugar composition analysis was carried out with a gas chromatograph GC-14B (Shimadzu Corporation, Kyoto, Japan) equipped with a flame ionization detector. The integrator was Chromatopac C-R8A (Shimadzu).
Table 1. Recovery of dietary fiber.*

\begin{tabular}{lc}
\hline \multicolumn{1}{c}{ Inedible parts of vegetables } & \% of dry weight \\
\hline Peel of taro & $68 \pm 4$ \\
Peel of Chinese yam & $19 \pm 2$ \\
Pea pod & $40 \pm 2$ \\
Broad bean pod & $57 \pm 4$ \\
Broad bean testa & $67 \pm 8$
\end{tabular}

* Values are mean \pm SD.

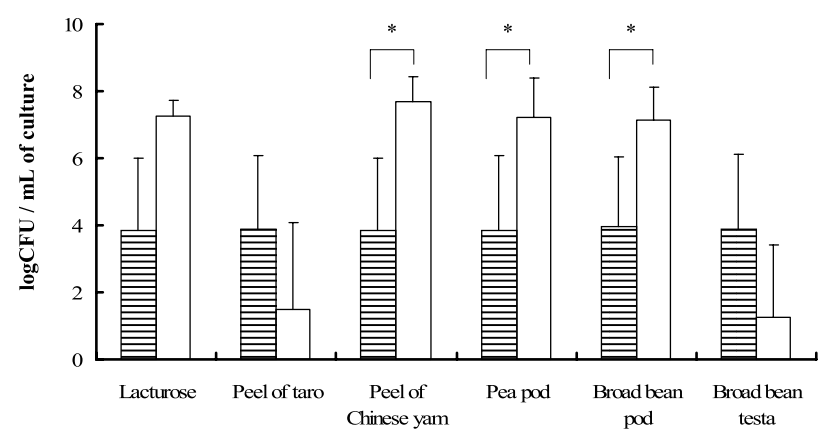

Fig. 1. Effect of dietary fibers on the growth of Bifidobacterium longum JCM1217. These media were cultured anaerobically at $37^{\circ} \mathrm{C}$. At $0 \mathrm{~h}($ 目) and after $48 \mathrm{~h}(\square)$, bacterial numbers were enumerated and depicted as $\log$ CFU (Colony Forming Unit). *Significantly different from the $0 \mathrm{~h}$ culture $(p<0.05)$ by $t$-test $(n=3)$.

The fused silica capillary column was $\mathrm{SP}^{\mathrm{TM}}-2380$ purchased from Supelco (Bellefonte, PA, USA), $30 \mathrm{~m} \times 0.25 \mathrm{~mm} \times 0.25 \mu \mathrm{m}$. The column temperature was programmed to rise from 190 to $235^{\circ} \mathrm{C}$ at $4^{\circ} \mathrm{C}$ per minute. The injector and detector temperatures were 240 and $290^{\circ} \mathrm{C}$, respectively. The flow rate of helium carrier gas was $1.0 \mathrm{~mL} / \mathrm{min}$ with a split ratio of $1: 50$. Each peak was identified by GC-MS (Agilent Technologies 5975C VL MSD equipped with 6890N Network GC System, USA).

Calculation and statistical analysis. All culture experiments were calculated in terms of means and standard deviations $(n=3)$. A $p$-value of less than 0.05 was considered to be significant. The statistical analysis was performed using SPSS version 17.0 for Windows (SPSS Inc., Tokyo, Japan).

\section{Results}

Recovery of dietary fiber

Recovery of DF is shown in Table 1. The weight of DF was depicted as \% of each dry weight. Peel of taro contained the highest amount of DF. The percentages of water-insoluble DF contained in DF preparations were 78.0 (peel of taro), 77.1 (peel of Chinese yam), 95.4 (pea pod), 86.2 (broad bean pod) and 83.2 (broad bean testa).

Screening of bifidogenic dietary fibers by pure culture of Bifidobacterium longum

Effect of DF on the growth of $B$. longum is shown in Fig. 1. After $48 \mathrm{~h}$ culture, the bacterial number of $B$. longum was significantly increased as compared with 
A

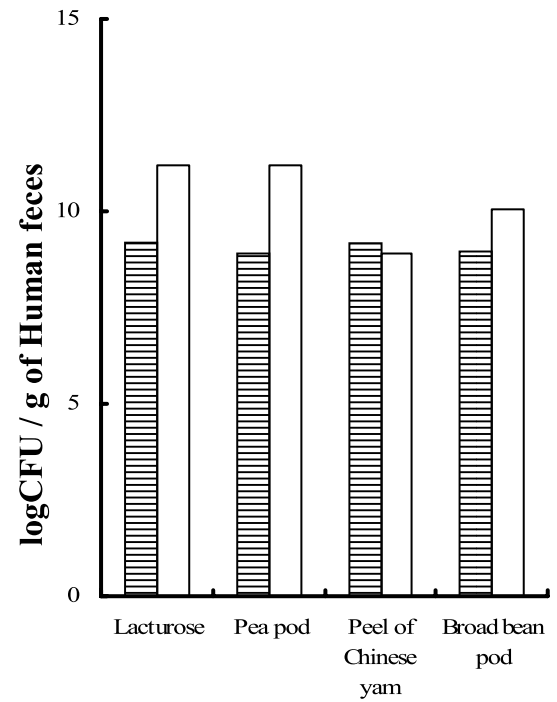

B

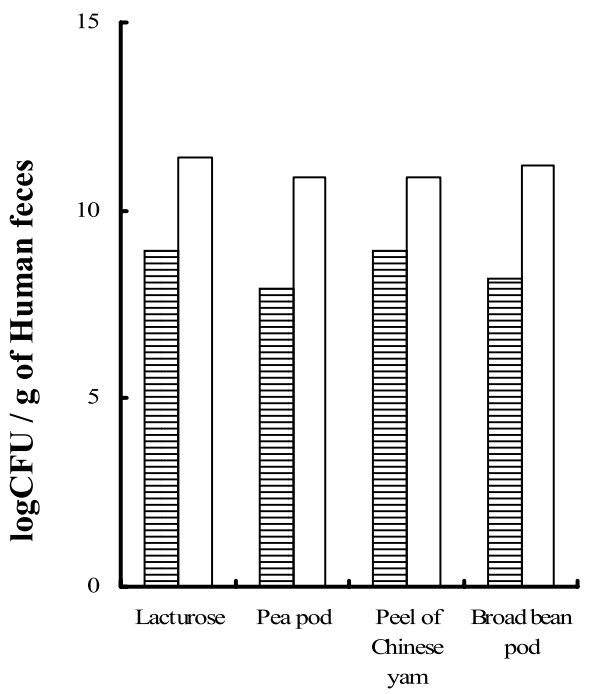

Fig. 2. Effect of dietary fibers on the growth of human fecal microbiota. These media were cultured anaerobically at $37^{\circ} \mathrm{C}$. At $0 \mathrm{~h}(\mathrm{E})$ and after $48 \mathrm{~h}(\square)$, bacterial numbers were enumerated and depicted as log CFU (Colony Forming Unit). A, bifidobacteria; B, lactobacilli.

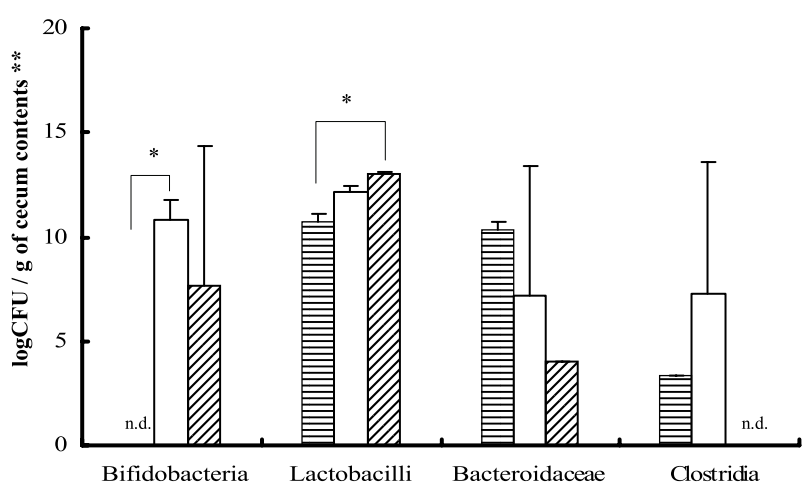

Fig. 3. Effect of dietary fiber extracted from pea pod on the growth of rat cecum microbiota. These media were cultured anaerobically at $37^{\circ} \mathrm{C}$. At $0 \mathrm{~h}($ 目) and after $48 \mathrm{~h}$ (lacturose: $\square$, dietary fiber: $\mathbb{Z}$ ), bacterial numbers were enumerated and depicted as log CFU (Colony Forming Unit)/g of cecum contents. * Significantly different from the $0 \mathrm{~h}$ culture $(p<0.05)$ by $t$-test $(n=3)$. ** Cecum contents were obtained from male Wistar rats, 8 wk old.

the number at $0 \mathrm{~h}$ in the DF from the peel of Chinese yam, pea pod, and broad bean pod. However, a decrease of bacterial number was observed in the DF from peel of taro and broad bean testa.

Fermentation studies of dietary fibers with human fecal or rat cecal microbiota

To detect the most bifidogenic DF, we used human fecal microbiota. After that, we used rat cecal microbiota. We determined how the bacterial numbers of bifidobacteria, lactobacilli, bacteroidaceae and clostridia in the medium were changed by fermentation with the DF.

Effect of DF on the growth of human fecal microbiota is shown in Fig. 2. The bacterial numbers of bifidobacteria and lactobacilli were most increased in the medium containing DF from pea pod (Fig. 2). As recovery of DF in pea pod indicated a moderate value among the inedible parts (Table 1), we selected pea pod as a candidate for prebiotics.

Effect of DF from pea pod on the growth of cecal microbiota of rat is shown in Fig. 3. Lactobacilli were significantly increased as compared with the number at $0 \mathrm{~h}$ in the medium containing DF. Bifidobacteria were not detected at $0 \mathrm{~h}$, but were observed at $48 \mathrm{~h}$ culture. That was not significant. As for lacturose, lactobacilli were increased as compared with the number at $0 \mathrm{~h}$. But that was not significant. Bifidobacteria were significantly increased. Bacteroidaceae and clostridia were decreased as compared with the number at $0 \mathrm{~h}$.

Analysis of constituent sugars

Sugar composition in DF is shown in Table 2. Each DF has xylose or mannose as major component. Ribose was only detected in peel of taro; fucose was detected except in peel of taro. Galactose and glucose were relatively high contents in peel of Chinese yam and broad bean testa, respectively.

\section{Discussion}

This is the first report related to the bifidogenic activity of inedible parts of vegetables as far as we know. We determined the bifidogenic properties of DF extracted from the inedible parts of vegetables, such as peel of taro, peel of Chinese yam, pea pod, broad bean pod and broad bean testa.

To determine the bifidogenic property of DF, an in vitro experiment was carried out with $B$. longum which was highly detected in human adults. The reason for using the rat cecal microbiota was that the number of bifidobacteria generated by feeding a normal cellulose diet was under the detection limit $(9,12)$. In our experiment, bifidobacteria were not detected at $0 \mathrm{~h}$ but were 
Table 2. Analysis of constituent sugars (\%).

\begin{tabular}{lrcccrrr}
\hline & Fucose & Ribose & Arabinose & Xylose & Mannose & Galactose & Glucose \\
\hline Peel of taro & n.d.* & 27.84 & 19.78 & 31.98 & 4.14 & 13.01 & 3.26 \\
Peel of Chinese yam & 4.10 & n.d. & 7.99 & 13.56 & 36.08 & 26.02 & 12.25 \\
Pea pod & 10.46 & n.d. & 6.71 & 65.31 & 5.16 & 7.15 & 5.21 \\
Broad bean pod & 0.28 & n.d. & 2.56 & 75.08 & 4.48 & 12.48 & 5.13 \\
Broad bean testa & 0.74 & n.d. & 3.75 & 57.45 & 4.22 & 8.35 & 25.48 \\
\hline
\end{tabular}

*n.d.: not detected.

increased after $48 \mathrm{~h}$ culture with the DF from pea pod. Lactobacilli were also increased after $48 \mathrm{~h}$ culture with that DF. The increasing rate of bifidobacteria and lactobacilli in that DF was comparable with lacturose. On the other hand, bacteroidaceae and clostridia were decreased after $48 \mathrm{~h}$ culture. From these results, it was shown that the DF from pea pod was a candidate for prebiotics.

As $5 \mathrm{~mL}$ of medium contained $0.5 \%$ of dietary fiber, the amount of DF was equivalent to $0.025 \mathrm{~g}$. This corresponded to $0.446 \mathrm{~g}$ of wet pea pod, which was calculated from the recovery of DF shown in Table 1. It was clear that a small amount of pea pod showed bifidogenic activity in vitro.

To differentiate the bifidogenic activity of DF, we carried out the sugar analysis of DF. We were interested in DF from peel of taro and broad bean testa, because the number of B. longum was decreased after $48 \mathrm{~h}$ culture. Peel of taro included a high content of ribose. Ribose was already detected in the DF of benniseed, pearl millet and quinoa (13). Broad bean testa included 5 times higher amounts of glucose than broad bean pod (Table 2 ). It is conceivable that B. longum could not hydrolyse DF containing ribose from peel of taro. Except for broad bean testa, the ratio of mannose, galactose, glucose was about $1: 1: 1$ or $1: 3: 1$. Too much glucose in broad bean testa may be the result of a cellulose-like structure and hinder the hydrolyzing enzyme of bifidobacteria. On the other hand, DF from pea pod, which was a candidate for prebiotics, had a different sugar composition. From the present results, xyloglucan was close to this DF. Xyloglucan has $\beta-1,4$ glucopyranose residue as a main chain and xylose attaches to the main chain. $\beta$-DGalactose, $\alpha$-L-fucose $(1 \rightarrow 2) \quad \beta$-D-galactose or arabinogalactose combine with the xylose chain (14). It was already reported that xyloglucan are fermented by gut microflora of rats (15). But it is not known whether xyloglucan is fermented by bifidobacteria. Further study to clarify the configuration of the sugar is needed.

\section{REFERENCES}

1) Gibson GR, Roberfroid MB. 1995. Dietary modulation of the human colonic microbiota: Introducing the concept of prebiotics. J Nutr 125: 1401-1412.

2) Jenkins D, Kendall CWC, Vuksan V. 1999. Inulin, oligofructose and intestinal function. J Nutr 120: 1431S1433 S.

3) Redgwell RJ, Fischer M. 2005. Dietary fiber as a versa- tile food component: an industrial perspective. Mol Nutr Food Res 49: 521-535.

4) Sembries S, Dongowski G, Jacobson G, Mehrländer K, Will F, Dietrich H. 2003. Effects of dietary fiber-rich juice colloids from apple pomace extraction juice on intestinal fermentation products and microbiota in rats. Br J Nutr 90: 607-615.

5) Manderson K, Pinart M, Tuohy KM, Grace WE, Hotchkiss AT, Widmer W, Yadhav MP, Gibson GR, Rastall RA. 2005. In vitro determination of prebiotic of oligosaccharides derived from an orange juice manufacturing by-product stream. Appl Environ Microbiol 71: 83838389.

6) Asano I, Nakamura Y, Hoshino H, Aoki K, Fujii S, Imura N, Iino H. 2001. Use of mannooligosaccharides from coffee mannan by intestinal bacteria. Nippon Nogeikagaku Kaishi 75: 1077-1083 (in Japanese).

7) Blaut B. 2002. Relationship of prebiotics and food to intestinal microflora. Eur J Nutr 4: 11-16.

8) Prosky L, Asp N-G, Furda I, DeVries JW, Schweizer TF, Harland BF. 1985. Determination of total dietary fiber in foods and food products: Collaborative study. J Assoc Off Anal Chem 58: 677-679.

9) Takamine K, Hotta H, Degawa Y, Moriura S, Kida K. 2004. Effect of dietary fiber prepared from sweet potato pulp on ceacal fermentation products and microflora in rats. Jpn Soc Appl Glycosci 52: 1-5.

10) Mitsuoka T, Ohno K, Benno Y, Suzuki K, Nanba K. 1976. The fecal flora of men. IV. Communication: Comparison of the newly developed method with the old conventional method for the analysis of intestinal flora. Zbl Bakt [Orig A] 234: 219-233.

11) Englyst H, Wiggins HS, Cummings JH. 1982. Determination of the non-starch polysaccharides in plant foods by gas-liquid chromatography of constituent sugars as alditol acetates. Analyst 107: 307-318.

12) Iwata E, Degawa Y, Sawaya $Y$, Takeyama A, Ookubo A, Yagi M, Hotta H, Benno Y. 2007. Effect of sucrose with L-arabinose on the number of bifidobacteria in the rat cecum. Eiyougaku Zasshi 65: 249-254 (in Japanese).

13) Oshodi AA, Ogungbenle HN, Oladimeji MO. 1999. Chemical composition, nutritionally valuable and functional properties of benniseed (Sesamum radiatum), pearl millet (Pennisetum typhoides) and quinoa (Chenopodium quinoa) flours. Int J Food Sci Nutr 50: 325-331.

14) Hayashi T. 1989. Xyloglucans in the primary cell wall. Annu Rev Plant Physiol Plant Mol Biol 40: 139-168.

15) Gray DF, Fry SC, Eastwood MA. 1993. Uniformly ${ }^{14} \mathrm{C}$ labelled plant cell walls: production, analysis and behavior in rat gastrointestinal tract. Br J Nutr 69: 177-188. 